\title{
A POSITIVE AND A NORMATIVE THEORY OF INCOME DISTRIBUTION
}

\author{
BY JAN TINBERGEN
}

Netherlands School of Economics

\begin{abstract}
A positive theory of income distribution based on assumptions concerning the supply of and demand for each type of productive service is presented. The demand function of the organizers of production may be derived from the maximization of profits with the income scale and the production function as restrictions. A normative theory based on the maximization of a social utility or welfare function is also considered. In the normative theory, production functions and balance equations (some representing compartmentalization of factor markets) are introduced as restrictions and again an income scale results, this time maximizing social welfare. Empirical testing is also considered. The positive theory was developed in part to take into consideration the fact that personal income distributions can reasonably well be described by log normal distributions, and that skill parameters are often normally distributed. Limited testing of the influence of wealth, intelligence, education, and sex suggest that these account for only a small part of the variance in the income distribution. This suggests the need for further research.
\end{abstract}

\section{Positive vs Normative Approach}

In this paper some theories of income distribution will be presented, which partially have already been published elsewhere by the author [5, 6]. Those published already were positive or analytical: their aim was to contribute to the explanation of (personal) income distribution. In the present paper I am going to add two versions of a normative theory, or a theory of the "optimal income distribution". The common element is the subject matter; we may look at incomes as the prices received for the supply of productive services, whether labour, land or capital. Since the largest share of national income goes to labour, the labour market will be included as the most important portion of the subject. In principle, however, an individual may supply a combination of the three factors of production. It is characteristic for reality and also for the theories to be presented that the labour market is compartmentalized: there are innumerable types of labour. An income distribution theory cannot therefore restrict itself to three compartments (for the three factors), but has to distinguish a considerably larger number of them.

A positive theory will be based on assumptions concerning the supply of and the demand for each type of productive service. Together the prices paid in the various compartments may be said to represent an income scale, possibly of many dimensions, relating the price of each type of service to its nature. Part of this scale may be called the wage scale, which under certain circumstances indicates the wage rate as a function of the parameters describing the type of labour. The same applies to the mixed service which a peasant or a shopkeeper supplies: partly labour of some type, partly capital. The individuals on the supply side of the market will determine their supply by maximizing their utility function, under the restrictions offered by the prevailing income scale. The 
individuals and institutions acting on the demand side will be called the organizers of production (in the widest sense). Their demand function may be derived from the maximization of profits with the income scale and the production function as restrictions. Alternatively, and following Cassel [1], we may formulate assumptions on the demand function directly without trying to dig more deeply. The income scale will be the unknown function of the problem; it will have to be such as to equalize supply and demand in all compartments.

A normative theory will be based on the maximization of a social utility or welfare function under a number of restrictions. Among the restrictions there will be one or more production functions and a number of balance equations, among them those of the compartments of the factor markets. Again an income scale will result, this time maximizing social welfare.

Both theories are part of a more comprehensive system describing the economy as a whole. In particular the normative theory will be part of welfare economics. Both theories will be influenced by assumptions made concerning other elements of the economy. In the positive theory, for instance, the tax system will be among the data. In the normative theory it will be among the unknowns, but even so enter into play: the solution of the general economic welfare problem also contains as an element the optimum tax system. Whereas the purpose and contents of the two theories are different, they make use of several common elements. We have already mentioned the production function(s) If the social welfare function is based on individual utility functions these too will appear in both theories. In our theories this will be so. Similarly the parameters used to characterize the various types of productive services are needed in both types of theory. So are the balance equations for all compartments. We will discuss some of these common foundations before offering some versions of both types of theory.

\section{Parameters Describing the Nature of Productive Services}

Although the theories cover all production factors, we will often use phrases derived from the labour market, mainly to keep our terminology simple and concrete. Each compartment of the factor market will be called a job and will be indicated by a parameter $s$. This may be a scalar or a vector. In the former case it is a figure out of one scale; in the latter case it is a set of figures. Practical examples from the labour market are the figures given in job evaluation. One or more of the components (if $s$ is a vector, or multidimensional) may be the quantity of private capital or of land the "job" requires. While job evaluation and the inclusion of other factors than labour require a multidimensional $s$, some of our examples will use-for simplicity's sake-a one-dimensional $s$. A fascinating example of such an $s$ is provided by Tuck [9] who defines it as the number of people the job concerned is supposed to supervise. Other examples sometimes used are the IQ required (Somermeyer [4]) or the number of years of training.

The nature of the productive service supplied may not only depend on the job description parameter $s$ but also on the skills of the person who takes the job. These we will indicate by $v$ (in Section 6 by $t$ ); this, then, is a personal parameter. 
Again it will be in principle multidimensional; but in some examples a onedimensional $v$ only will be used. If we admit many dimensions, several of these will be of the same nature as the corresponding dimensions of $s$. Some of the components may not have their counterpart in a component of $s$; thus, the intensity or speed of work may be a personal parameter only. As a rule the components of $s$ and $v$ which are of the same nature will not have very different values. A job requiring a high IQ will not be taken by or given to a person having a very low IQ. On some occasions we will speak of $s$ as the skills required and of $v$ as the skills available.

\section{Nature of Utility Functions}

In the presentation of our theories we will act as if utility is measurable. This does not mean that all our conclusions depend on that procedure. Several of our arguments can be easily reformulated so as to be independent of the procedure. Some forms of non-measurability can be translated to mean that only the ratios between some coefficients can be observed instead of these coefficients themselves.

Independently of the preceding remarks two questions are of paramount importance. These we will now discuss in succession. The first question is what variables occur in a utility function. The answer is that there are three categories, namely:

(a) quantities consumed of all goods and services;

(b) parameters representing the nature of the job taken;

(c) parameters representing the nature of the individual.

The third category can be subdivided into those indicating the skills of the individual and those indicating the needs of the individual or his family.

In our examples we will not distinguish between various types of goods and take only one variable, real income after tax, to represent category (a). We already discussed category (b) and will take our parameter $s$ to represent it. When multidimensional, $s$ stands for a series of variables. We also already discussed the skill parameter $v$. As examples of the parameters indicating the needs of the individual we consider, on the one hand, $s$ and $v$, but on the other hand a new parameter $n$, standing for family size. It is perfectly conceivable to include either in the $v$ or separately the health of the individual which will co-determine his needs.

The second question to be discussed is in what way account should be taken of the differences existing between various individuals. The way that will be followed in this paper is to assume that the types of parameters just discussed include the possibility of taking into account any differences whatsoever. As a consequence it will not be necessary to assume different values of the coefficients occurring in the utility function. These, then, will be taken equal for all individuals and they may be seen as the expression of an ethical viewpoint of the "fundamental equality of all men". Alternatively this viewpoint may be expressed as follows: it is assumed that in every respect all men are the same except where observation shows deviations. These observations will then be used to estimate 
the values of parameters of category (c). It goes without saying that, moreover, only differences have to be considered which are essential to our problem. Clearly the colour of skin, hair or eyes or the form of the nose do not matter. Even the preference for Swiss cheese or American cheese does not seem to be relevant.

For the specifications of the individual utility functions used in my examples I refer to Sections 6-8.

\section{Nature of Production Functions or Demand Functions}

We will not use production functions in all the versions of our theory. When they are used, however, it is essential that the nature of the jobs and the skills of those who take them will influence the product obtained, that is, production functions using only one type of labour are of very limited value, Several or even many types will have to be distinguished. At the same time, the numbers of individuals taking the various jobs and showing various skills must enter into the production function. In the most sophisticated approach the numbers of individuals showing specified values of both the nature of the job $s$ and the level of the skills $v$ will be among the variables in the production function. In less elaborate versions only the job indicator will be used. There is not much scope for also considering the length of the working day since this is rarely changed and even more rarely a free choice of the individual.

In models where not the production function but only the demand functions for various types of labour are used, again several or even many types of jobs $s$ have to be introduced if a minimum of realism is required. The demand functions may be rigid or they may depend on the income (or wage) scale; for details of the assumptions made the reader is referrred to Sections 6-8.

\section{Remarks on Mathematical Techniques}

It is self-evident and has already been stressed in the previous sections that the numbers (or "frequencies") of individuals showing up in each compartment of the factor market system - on the supply side and on the demand side-play a central role in any theory of income distribution. In the examples to be discussed two mathematical techniques have been used to cope with these frequencies, namely discrete and continuous frequency distributions. In the former case a finite number of $s$ or $v$ values or both are distinguished and the numbers of individuals involved are finite too. This remains so if some parameter of category (c) of Section 3 has been added also. An example will be found in Section 7. In this approach the true variables of the problem are these frequencies $\varphi_{s v n}$. In the latter case, where continuous frequency distributions are used, the job and skill parameters are permitted to take any value over a closed or open interval and the frequency distribution is assumed to be of some regular type. The advantage of this method is that an infinite number of compartments can be considered and yet the number of unknowns be kept limited; typically the unknowns are now the parameters of the frequency distribution, such as the average, the standard deviation and so on. In our example we restrict ourselves to normal 
distributions where only a number of correlation coefficients have to be added to the distribution parameters already mentioned if the distribution has two or more dimensions. In more complicated distributions higher moments may occur.

While this approach permits a very elegant treatment of some aspects of income distribution (cf. Sections 6 and 8), the disadvantage of it clearly is that we impose a strait-jacket on reality-for instance that the distributions are normal-which may not always be admissible and has to be checked against observations.

\section{Some Versions of a Positive Theory}

In earlier publications $[5,6,8]$ I have offered a positive theory of income distribution showing the following characteristics in terms of the concepts discussed in the previous sections.

(a) There are no direct taxes.

(b) The job parameter $s$ is two-dimensional, implying that it has two components $s_{1}$ and $s_{2}$.

(c) The skill parameter $t$ has two dimensions also, $t_{1}$ and $t_{2}$, corresponding with the $s$-components.

(d) No parameters representing needs have been introduced.

(e) Normal frequency distributions for the job parameters and for the skill parameters have been assumed to be given and characterized by $\bar{s}_{1}, \bar{s}_{2}$, $\sigma_{1}, \sigma_{2}$ and $r_{s}$ for the former and by $\bar{t}_{1}, \bar{t}_{2}, \tau_{1}, \tau_{2}$ and $r_{t}$ for the latter.

(f) Individual utility functions are used of the shape

$$
\omega=\omega_{0} l n l-\frac{1}{2} \omega_{1}\left(s_{1}-t_{1}\right)^{2}-\frac{1}{2} \omega_{2}\left(s_{2}-t_{2}\right)^{2}
$$

where (real) income is $l$ and $\omega_{0}, \omega_{1}$ and $\omega_{2}$ are the same for all individuals. The last two terms indicate the compensation the individual desires if he has to take a job $s$ not coinciding with this skills $t$. By it he experiences a dissatisfaction only accepted if compensated for. This dissatisfaction is the same for positive and for negative values of the "tension" $s-t$. Generally the tension will not be large.

The unknown of the problem is the income scale:

$$
\ln l=\lambda\left(s_{1}, s_{2}\right)=\lambda_{00}+\lambda_{10} s_{1}+\lambda_{01} s_{2}+\frac{1}{2} \lambda_{20} s_{1}{ }^{2}+\lambda_{11} s_{1} s_{2}+\frac{1}{2} \lambda_{02} s_{2}{ }^{2}
$$

tentatively assumed to be of quadratic form. This assumption was tested and shown to be admissible and just sufficient to solve the problem.

In the frequency distributions for $s$ and $t, r_{s}$ and $r_{t}$ are the correlation coefficients between $s_{1}$ and $s_{2}$ on the one hand and between $t_{1}$ and $t_{2}$ on the other hand. Without loss of generality it can be assumed that $\tau_{1}=\tau_{2}=1$ and $r_{t}=0$, since the choice of the skill criteria can be such that the two are not correlated and their measurement be such that the standard deviations are 1 . 
It appears that demand and supply in each compartment of the factor market can be equalized if the coefficients satisfy the following conditions:

$$
\begin{aligned}
& \bar{t}_{1}=\left(1-\omega_{0} \lambda_{20}\right) \bar{s}_{1}-\omega_{0}^{\prime} \lambda_{11} \bar{s}_{2}-\omega_{0} \lambda_{10} \\
& \bar{t}_{2}=-\omega_{0}^{\prime \prime} \lambda_{11} \bar{s}_{1}+\left(1-\omega_{0}^{\prime \prime} \lambda_{02}\right) \bar{s}_{2}-\omega_{0}^{\prime \prime} \lambda_{01} \\
& \left(1-\omega_{0} \lambda_{20}\right)^{2}=\left(\frac{1}{\sigma_{1}{ }^{2}}-\frac{\omega_{2} r_{s}{ }^{2}}{\omega_{1}{ }^{2} \sigma_{1}{ }^{2}+\omega_{2}{ }^{2} \sigma_{2}{ }^{2} \pm 2 \omega_{1} \omega_{2} \sigma_{1} \sigma_{2} \sqrt{1-r_{s}{ }^{2}}}\right) \frac{1}{1-r_{s}{ }^{2}} \\
& \omega_{0}^{\prime} \lambda_{11}^{2}=\frac{\omega_{2} r_{s}^{2}}{\omega_{1}^{2} \sigma_{1}+\omega_{2}^{2}{\sigma_{2}}^{2} \pm 2 \omega_{1} \omega_{2} \sigma_{1} \sigma_{2} \sqrt{1-r_{s}^{2}}} \frac{1}{1-r_{s}^{2}}
\end{aligned}
$$

and a formula for $\lambda_{02}$ symmetrical to the one for $\lambda_{20}$, where

$$
\omega_{0}^{\prime}=\frac{\omega_{0}}{\omega_{1}} \text { and } \omega_{0}^{\prime \prime}=\frac{\omega_{0}}{\omega_{2}} .
$$

These formulae are somewhat complicated. Their meaning becomes clearer if we note that $\lambda_{11}$ depends heavily on $r_{s}$ and that for $r_{s}=0$ we have:

$$
\begin{array}{r}
\lambda_{10}=\frac{\omega_{1}}{\omega_{0}}\left(\frac{\bar{s}_{1}}{\sigma_{1}}-\frac{\tilde{t}_{1}}{\tau_{1}}\right) ; \quad \lambda_{01}=\frac{\omega_{2}}{\omega_{0}}\left(\frac{\bar{s}_{2}}{\sigma_{2}}-\frac{\bar{t}_{2}}{\tau_{2}}\right) \\
\lambda_{20}=\frac{\omega_{1}}{\omega_{0}}\left(1-\frac{\tau_{1}}{\sigma_{1}}\right) ; \quad \lambda_{11}=0 ; \quad \lambda_{02}=\frac{\omega_{2}}{\omega_{3}}\left(1-\frac{\tau_{2}}{\sigma_{2}}\right)
\end{array}
$$

where we have given the values valid also if $\tau_{1}$ and $\tau_{2} \neq 1$.

The following conclusions can be drawn: A linear formula for the income scale $\lambda\left(s_{1}, s_{2}\right)$, that is $\lambda_{20}=\lambda_{11}=\lambda_{02}=0$ is sufficient if (i) $r_{s}=0$; (ii) $\tau_{1}=\sigma_{1}$ and (iii) $\tau_{2}=\sigma_{2}$. These conditions imply that the frequency distribution of the jobs offered does not show correlation between $s_{1}$ and $s_{2}$ and has the same standard deviations as the frequency distribution of the skills. If the standard deviations do not coincide we see that quadratic terms are needed, that is, a more than proportional increase in $\ln l$ if the skill distribution has smaller standard deviations than the job distribution. In simple language the individuals with more than average skill have to be encouraged by higher pay in order to take the "difficult" jobs; higher pay, that is, than if the standard deviations were equal. The inverse is true if the skill distribution shows a higher standard deviation than the job distribution. The mixed term in $\lambda\left(s_{1}, s_{2}\right)$, that is the term $\lambda_{11} s_{1} s_{2}$, is needed only if there is correlation between the job requirements for $s_{1}$ and $s_{2}$. This means that, with a positive correlation, standing for a higher-than-otherwise demand for people with high skills on both counts, this combination must be paid additionally, because these people are scarce in comparison to the numbers required. The linear terms indicate that "higher" jobs must be paid more than "lower", if the average skill required surpasses the average skill available in the population, both measured in terms of the standard deviations (cf. formulae 6.7); in the opposite case they must be paid less than "lower" jobs.

All this can be summarized somewhat loosely by saying that incomes paid must reflect the relative scarcity, that is, the scarcity of skills available in comparison to the required skills. It implies, and this is not always realized, that equal incomes are possible not merely if all people are equally skilled-which they 
evidently are not-but already if only the skill distribution required by the organizers of production coincides with the actual skill distribution.

Several of the assumptions made can be released in order to formulate a theory of more general validity. Some remarks will be made here, classified by using the same letters as in the beginning of this section.

(a) It is possible to introduce taxes; this will be shown in Section 8 for the normative version, but it is equally applicable to the positive version.

(b) As far as I can see the number of dimensions of the skill requirements and the skills available, can be increased without changing the nature of the treatment. It will become rapidly more complicated with an increasing number of dimensions, however.

(c) Apart from the parameters $v$ corresponding with components of $s$ there may be other personal parameters, as already stated in Section 2, for instance, work speed or intensity, An example of the inclusion of such parameters has been presented elsewhere [8] and a similar one will be given in Section 8.

(d) Examples of the inclusion of need parameters will be given in Sections 7 and 8 .

(e) It is not necessary to assume that the demand frequency distribution (that of $s$ ) is given independently from the income scale. As shown in the original publication some types of reaction of demand to that scale can be introduced without changing the method. But clearly the results become somewhat more complicated.

Other than normal frequency distributions will be discussed for simple cases only, again in Sections 7 and 8 . Since the (log)-normal frequency distribution covers large portions of the job and skill distribution, however, a limitation to normal distributions does not seem to be a serious inconvenience. It has to be admitted, however, that for the element of personal capital other distributions are more adequate; see, however [10].

(f) Admittedly also the utility function is of a very simple type. Here some more general shapes will again be discussed in Sections 7 and 8 .

\section{A Normative Theory with Discrete Frequency Distribution}

In the present section I will discuss a version of a normative theory with the following characteristics, listed similarly to what was said in Section 6.

(a) A tax system is assumed to exist making it possible that income after $\operatorname{tax} x_{s v n}$ does not coincide with the factor payment received by the individual considered.

(b) The job parameter $s$ is one-dimensional and in our example even takes only two different values, 1 and 2 .

(c) The skill parameter $v$ is dealt with in the same way.

(d) The individual is supposed to have needs depending on $s$ and $v$ and moreover on family size $n$, which again in our example takes only two different values, 1 and 2 . The needs resulting from $s$ and $v$ are represented 
by $c_{0} v+c_{1} s$ and might be called "professional costs". The way the needs of the family are taken care of will be discussed below, under (f).

(e) The frequency of any combination $(s, v, n)$ is written $\varphi_{s u n}$; no a priori assumptions are made on the nature of this (discrete) frequency distribution. Since $v$ and $n$ are supposed to be given for each income earner (head of family), we assume the values of their frequencies $F_{v n}$ as given. We will also use the notation $\varphi_{s v}=\varphi_{s v 1}+\varphi_{s v 2}$ for the number of individuals with skill $v$ taking a job $s$.

(f) The individual utility function for a family head $\omega_{s v n}$ is given the form:

$$
\omega_{s v n}=n \ln \frac{x_{s v n}-c_{0} v-c_{1} s-c_{2}(s-v)^{2}}{n} .
$$

Here $c_{2}(s-v)^{2}$ stands for the compensation the family head desires if he has to take a job $s$ not coinciding with his skills $v$. After deducting from his income-after-tax $x$ both his professional costs and his compensation, he is left with the amount $x^{\prime}$ he and his family are deriving their satisfaction from, which per adult unit is assumed to be $\ln \left(x^{\prime} / n\right)$. The total satisfaction of the family is then $n$ times this amount, as indicated in (7.1).

(g) A production function is assumed in the Cobb-Douglas tradition, written

$$
y=k^{\kappa} \varphi_{11}{ }^{\rho_{11}} \varphi_{12}{ }^{\rho_{12}} \varphi_{21}{ }^{\rho_{12}} \varphi_{22}{ }^{\rho_{22}}
$$

where $k$ is the capital stock, supposed to be publicly owned. We assume that $\kappa+\rho_{11}+\rho_{12}+\rho_{21}+\rho_{22}=1$ or that $y$ is linear homogeneous.

The theory will be formulated in the usual welfare economic fashion, where the problem is to maximize social welfare $\Omega$ under a number of restrictions.

We define social welfare as the sum of individual utilities:

$$
\Omega=\sum_{i} \sum_{s} \sum_{v} \sum_{n} \varphi_{s u n} x_{s v n}
$$

where for simplicity's sake we have omitted the time index $t$ from the symbols so far introduced. This will appear possible in this very simple model for reasons set out elsewhere [7] and become clear also in what follows.

The restrictions are:

(i) the production function (7.2);

(ii) a balance equation for each time period $t$ :

$$
y-\sum_{t} \sum_{v} \sum_{n} \varphi_{s v n} x_{s v n}-k_{t}+k_{t-1}=0 .
$$

where $k_{t}$ is the capital stock at the end of period $t$;

(iii) four balance equations

$$
\sum_{s} \varphi_{s v n}-F_{v n}=0
$$

(iv) and four balance equations:

$$
\varphi_{s v}-\sum_{n} \varphi_{s v n}=0 .
$$


Using the method of Lagrange multipliers $\lambda^{\prime}, \lambda, \mu_{v n}$ and $\nu_{s v}$, we have then to maximize (summed over time):

$$
\begin{aligned}
& \sum_{s} \sum_{v} \sum_{n} \varphi_{s v n} n l n \frac{x_{s v n}-c_{0} v-c_{1} s-c_{2}(s-v) \bar{s}}{n} \\
& +\lambda^{\prime}\left(-y+k_{t-1} \varphi_{11}^{\rho_{11}} \varphi_{12}^{\rho_{12}} \varphi_{21}{ }^{\rho_{21}} \varphi_{22}{ }^{\rho_{22}}\right)+\lambda\left(y-\sum_{s} \sum_{v} \sum_{n} \varphi_{s v n} x_{s v n}-k_{t}+k_{t-1}\right) \\
& +\mu_{v n}\left(\sum_{s} \varphi_{s v n}-F_{v n}\right)+v_{s v}\left(\varphi_{s v}-\sum_{n} \varphi_{s v n}\right)
\end{aligned}
$$

In this expression all symbols without a time index should carry $t$. It will be observed that production in time period $t$ is assumed to use the capital stock of $t-1$. As is well known we obtain the optimum conditions by consecutive differentiation of (7.7) with respect to each of the variables occurring there; thus we have as many equations as variables. The equations contain the Lagrange multipliers as further unknowns but we also have all the restrictions as additional equations and these are equal in number to the number of multipliers. The conditions we find are:

$$
\varphi_{s v n} \frac{n}{x_{s v n}-c_{0} v-c_{1} s-c_{2}(s-v)^{2}}-\lambda \varphi_{s v n}=0
$$

or

$$
\begin{gathered}
{\frac{x_{s v n}-c_{0} v-c_{1} s-c_{2}(s-v)^{2}}{n}}_{n}^{2}=\frac{1}{\lambda} \\
\omega_{s v n}-\lambda x_{s v n}+\mu_{v n}-v_{s v}=0 \\
\lambda^{\prime} \rho_{s v} \frac{y}{\varphi_{s v}}+v_{s v}=0 \\
-\lambda^{\prime}+\lambda=0 \\
-\lambda_{t}+\lambda_{t+1}+\lambda_{t+1}^{\prime} \kappa \frac{y_{t+1}}{k_{t}}=0
\end{gathered}
$$

In order to interpret the implications of the solution we need not solve explicitly the system of equations. The following statements can be made, however, which clarify the nature of the optimum.

(A) Equations I tell us that marginal utilities must be equal for all value combinations of $s, v$ and $n$ (since $\lambda$ is independent from these values).

(B) Equation (IV) shows that $\lambda^{\prime}=\lambda$.

(C) By (V) we can now express $\lambda_{t}$ in terms of $\lambda_{t-1}, k_{t-1}$ and $y_{t}$, which itself can be expressed with the aid of (7.2) in terms of $k_{t-1}$ and the $\varphi_{s v}$.

(D) From the fact that $v_{s v}$ is the multiplier for the supply of labour in job $s$ with skill $v$ and that $\lambda$ is the multiplier for production, we can derive that $-\left(\nu_{s v} / \lambda\right)$ equals the real income payment to factor $(s, v)$ and from (III) we find that this real income (before tax) $l_{s v}$ equals its marginal 
productivity:

$$
l_{s v}=\rho_{s v} \frac{y}{\varphi_{s v}}
$$

(E) We can eliminate the multiplier $\mu_{v n}$ by taking the two equations (II) referring to the same $(v, n)$ :

$$
0=\omega_{1 v n}-\lambda x_{1 v n}-\nu_{1 v}=\omega_{2 v n}-\lambda x_{2 v n}-\nu_{2 v} .
$$

Since all $\omega_{s v n}=n \ln (1 / \lambda)$ (from (7.1) and (I)) we can leave out from the second equation the $\omega$-term. Moreover, by (I) we have:

and:

$$
x_{1 v n}=c_{0} v+c_{1}+c_{2}(1-v)^{2}+\frac{n}{\lambda}
$$

$$
x_{2 v n}=c_{0} v+2 c_{1}+c_{2}(2-v)^{2}+\frac{n}{\lambda}
$$

Dividing through by $\lambda$ and leaving out equal terms on both sides of (7.9) we are left with equations for which $v=1$ and $v=2$ run:

$$
\begin{aligned}
& l_{11}=l_{21}-c_{1}-c_{2} \\
& l_{12}=l_{22}-c_{1}+c_{2}
\end{aligned}
$$

These express that factor payments to individuals with the same skill but in different jobs must differ by the difference in professional costs and the compensation for "tension" connected with the different jobs they take.

The remainder of the solution is of secondary importance. It appears that in this version the equations are partly dependent which enables us to choose freely four of the $\varphi_{s v n}$, for instance those referring to one family size in each of the job-skill combinations $(s, v)$.

This version of a normative theory on income distribution shows some features which are characteristic of many other welfare economic models, namely the simultaneous fulfilment of (I) and (III), that is marginal utility equalization among all individuals and factor payments depending on the marginal productivity of each job-skill combination. If one likes, they could be formulated as "to everybody according to his needs and from everybody according to his skills", the well-known formulation of the communist phase in development.

The main problem of implementation is whether a tax system can be defined which links these two principles. In welfare economics it is known as the lumpsum tax system, which does not tax marginal transactions. It seems worth while to inquire what data the tax depends on. Writing $t_{\text {svn }}$ for the tax to be paid, we find:

$$
t_{s v n}=l_{s v}-x_{s v n}=l_{s v}-c_{0} v-c_{1} s-c_{2}(s-v)^{2}-\frac{n}{\lambda}
$$

Since the expression $l_{s v}-c_{0} v-c_{1} s-c_{2}(s-v)^{2}$ is independent from $s$, we find that $t_{s v n}$ depends on $v$ and $n$ only. It does not therefore affect the choice of the job, but depends on the individual's skills and needs. 
While this version of the normative theory had been kept overly simple in order to illustrate some principles, it will be possible to introduce several generalizations. Using the same letters as in the beginning of this section we may mention the following possibilities:

(a) There is no need to generalize here.

(b), (c), (d) The number of classes for each of the parameters $s, v$ and $n$ can be chosen larger.

(e) Here again no need for generalization exists (cf. however, Section 8).

(f) Clearly utility functions, both individual and social, remain a large domain for further research, so large indeed that separate research projects will be needed.

(g) In the field of production functions a considerable amount of research going beyond the Cobb-Douglas function is already available. Thus the C.E.S. functions as well as the functions containing vintages of capital goods have already been adapted to empirical material, especially by Solow [3] and several others. Functions using various types of labour have been presented in forms somewhat different from the one chosen here, namely by the introduction of a "volume of education", as, for instance, by Denison [2]. Moreover, the process of education may itself be introduced as a "production process" or even several of them [7].

(h) Finally, private capital may be introduced as one of the components of both $s$ (job requirement) and $v$ (skills, albeit of an institutional character).

\section{A Normative Theory with Continuous Frequency}

\section{Distributions}

As a third example of a theory of income distribution, but again a normative one, a version will be offered using continuous frequency distributions as in Section 6, but also containing a tax system as in Section 7. The characteristics are:

(a) A one-dimensional job parameter $s$ is assumed to suffice, where $s$ can vary continuously.

(b) A one-dimensional skill parameter $v$ is assumed, also able to change continuously.

(c) In addition a parameter for work intensity $v_{2}$ and the family size $n$ will be introduced.

(d) An income scale will be tentatively assumed in the form:

$$
l\left(s, v_{2}\right)=\lambda_{0}+\lambda_{1} s+\lambda_{2} v_{2}+\frac{1}{2} \lambda_{3} s^{2}+\frac{1}{2} \lambda_{4} v_{2}^{2}
$$

(e) A tax depending on $v$ only will be assumed:

$$
t(v)=\tau_{0}+\tau_{1} v+\tau_{2} v^{2}
$$

(f) Individual utility functions are assumed to be

$$
\omega=\omega_{0} n \ln \frac{l\left(s, v_{2}\right)-t(v)}{n}-\frac{1}{2} \omega_{1}(s-v)^{2}-\omega_{2} v_{2}-\frac{1}{2} \omega_{3} v_{2}^{2}
$$


(g) Social utility $\Omega=\Sigma \omega$ over all individuals.

The present version of a normative theory will be sketched out only as far as relevant to the determination of the distributions of incomes both before and after tax. The sketch will be based on results found with the aid of more complete models of a welfare optimum from which the following propositions will be taken over:

(i) In the optimum each individual chooses his job and his work intensity by seeking maximum $\omega$ with given income and tax scales;

(ii) In the optimum the tax system must be such as to equalize marginal utilities among individuals;

In mathematical symbols this requires:

$$
\text { (i): } \frac{\partial \omega}{\partial s}=0 \quad \text { and } \frac{\partial \omega}{\partial v_{2}}=0 ; \quad \text { (ii): } \frac{\partial \omega}{\partial l}=\lambda
$$

With the aid of the scales tentatively assumed (8.4) becomes:

$$
\begin{aligned}
& \frac{\partial \omega}{\partial s}=\frac{\partial \omega}{\partial l} \frac{\partial l}{\partial s}-\omega_{1}(s-v)=\frac{\partial \omega}{\partial l}\left(\lambda_{1}+\lambda_{3} s\right)-\omega_{1}(s-v)=0 \\
& \frac{\partial \omega}{\partial v_{2}}=\frac{\partial \omega}{\partial l} \frac{\partial l}{\partial v_{2}}-\omega_{2}-\omega_{3} v_{2}=\frac{\partial \omega}{\partial l}\left(\lambda_{2}+\lambda_{4} v_{2}\right)-\omega_{2}-\omega_{3} v_{2}=0 \\
& \frac{\partial \omega}{\partial l}=\frac{\omega_{0} n}{\lambda_{0}+\lambda_{1} s+\lambda_{2} v_{2}+\frac{1}{2} \lambda_{3} s^{2}+\frac{1}{2} \lambda_{4} v_{2}^{2}-\tau_{0}-\tau_{1} v-\tau_{2} v^{2}}=\lambda
\end{aligned}
$$

From (8.5) and (8.6) we find the "job choice"

$$
\text { "job choice" } \quad s=\frac{\lambda \lambda_{1}+\omega_{1} v}{\omega_{1}-\lambda \lambda_{3}}
$$

and the

$$
\text { "intensity choice" } v_{2}=\frac{\lambda \lambda_{2}-\omega_{2}}{\omega_{3}-\lambda \lambda_{4}}
$$

Upon substituting these values into (8.7) we find the values of the tax parameters $\tau_{0}, \tau_{1}$ and $\tau_{2}$ which make (8.7) an identity. They are:

$$
\begin{aligned}
\tau_{0}= & -\frac{\omega_{0} n}{\lambda}+\lambda_{0}+\frac{\lambda \lambda_{1}{ }^{2}}{\omega_{1}-\lambda \lambda_{3}}+\lambda_{2} \frac{-\omega_{2}+\lambda \lambda_{2}}{\omega_{3}-\lambda \lambda_{4}}+\frac{1}{2} \lambda_{3}\left(\frac{\lambda \lambda_{1}}{\omega_{1}-\lambda \lambda_{3}}\right)^{2} \\
& +\frac{1}{2} \lambda_{4}\left(\frac{-\omega_{2}+\lambda \lambda_{2}}{\omega_{3}-\lambda \lambda_{4}}\right)^{2} \\
\tau_{1}= & \frac{\lambda_{1} \omega_{1}}{\omega_{1}-\lambda_{1} \lambda_{3}}+\frac{\lambda \lambda_{1} \lambda_{3} \omega_{1}}{\left(\omega_{1}-\lambda \lambda_{3}\right)^{2}} \\
\tau_{2}= & \frac{1}{2} \lambda_{3} \frac{\omega_{1}{ }^{2}}{\left(\omega_{1}-\lambda \lambda_{3}\right)^{2}} .
\end{aligned}
$$

The tax will depend negatively on family size $n$ and positively on skill $v$ and $v^{2}$, if $\omega_{1}>\lambda \lambda_{3}$, which is probable: the expression (8.8) for $s$ suggests this. 
As in Section 6 (but now in a simpler way, since we took $s$ and $v$ onedimensional only) we can use coefficients $\lambda_{1}$ and $\lambda_{3}$ to adapt the frequency distribution of $s$ to that of $v$, meaning that for each value of $s$, corresponding to a value of $v$ according to (8.8.) the frequency density is equal to that of the $v$ considered. This is equivalent to saying that there is equality of demand and supply in each compartment of the factor market. If both distributions are normal, this adaptation is possible indeed. It will also be possible for other two-parameter distributions, for instance for a constant-density distribution over a finite interval.

Coefficients $\lambda_{2}$ and $\lambda_{4}$ can be determined by the organizers of production so as to attain a production optimum, for instance maximum profits.

The most important features of this sketch are the following:

(i) Equilibrium in all compartments of the factor market can be obtained by a proper choice of the income scale. Since employers will only employ individuals if their income equals at least their productivity, this income scale reflects marginal productivities.

(ii) Incentives for the choice of jobs and of intensity of work are not affected by the tax system here considered: the tax system does not depend on either $s$ or $v_{2}$.

(iii) Yet the tax system here devised results in incomes after tax with the same marginal utilities for all, that is "according to needs".

(iv) While the model in Section 7 only contained two values of $s, v$ and $n$, as many values as desired can be distinguished in the present model.

Some generalizations will be desirable and possible; among them the introduction of a multidimensional $s$ and $v$.

\section{EMPIRICAL Testing}

Theories can only be accepted after having been tested. Although the present paper was supposed to present theories only, some remarks on their testing are desirable. Strictly speaking only a "positive" theory claims to portray reality and this section therefore mainly deals with the theory presented in Section 6 and its predecessors. Normative theories contain elements which must be tested, but as such they are policy devices, useful only as far as their assumptions have been tested.

The theory presented in Section 6 was constructed in order to fit at least one feature of reality which had been brought out by observation before [10], namely that personal income distributions can be reasonably well described by the log-normal distribution. In addition it is based on the assumption that skill parameters can often by presented as normally distributed. Finally it tried to introduce the possibility of absorbing the rich material which job evaluation and the corresponding evaluation of persons have made available in recent decades.

Even so it should be noted that one elaborate test undertaken by Somermeyer [4] throws doubt on the theory or at least raises some serious questions. Using the method of analysis of variance and applying it to a number of geographical subdivisions of the Netherlands this author estimates the influence of 
personal wealth, intelligence and education, sex and age and degree of economic activity, basing the analysis on a linear regression equation and using material for one single year. His main finding is that a large random component is left (71 per cent) after the estimation of the variance due to the various factors. The influence found for the only skill parameter at his disposal, namely IQ, is limited to 7 per cent. This may be due to the fact that as a measure of skill IQ is not very representative. The large random factor may represent also windfall profits, which in one single year may be considerable. There is scope for extended research here, including the number of years of education, whose influence has been found to be considerable by many authors. Moreover, some of the elements used in both the positive and the normative theories can be tested directly; among them production functions using various types of labour as their arguments and utility functions. Especially the latter have been the subject of very little empirical research. It would require another paper to deal with the patricular types of problems one encounters in this field.

\section{REFERENCES}

[1] G. Cassel, Theoretische Sozialökomonie, Leipzig 1918.

[2] E. F. Denison, Why Growth Rates Differ, New York 1967.

[3] R. M. Solow, Capital Theory and the Rate of Return, Amsterdam, 1963.

[4] W. H. Somermeyer, "An Analysis of Income Variance in the Netherlands", Statist. Informationen, 1967, p. 81 .

[5] J. Tinbergen, "On the Theory of Income Distribution", Weltwirtschaftliches Archiv, 77 (1956), p. 155.

[6] J. Tinbergen, Selected Papers, Amsterdam 1959.

[7] J. Tinbergen, "Over het dynamische welvaartsmaximum", (Dutch), Meded. der Kon. Ned. Akad, van Wetensch., afd. Letterk., Nieuwe reeks, deel 28, no. 4.

[8] J. Tinbergen, Economic Policy: Principles and Design, Amsterdam 1956.

[9] R. H. Tuck, An Essay on the Economic Theory of Rank, Oxford 1954.

[10] J. van der Wijk, Inkomens- en vermogensverdeling (Dutch, with English summary), Haarlem 1939. 
Copyright of Review of Income \& Wealth is the property of Blackwell Publishing Limited. The copyright in an individual article may be maintained by the author in certain cases. Content may not be copied or emailed to multiple sites or posted to a listserv without the copyright holder's express written permission. However, users may print, download, or email articles for individual use. 